\title{
Existence for boundary value problems of two-term Caputo fractional differential equations
}

\author{
Badawi Hamza Elbadawi Ibrahim, Qixiang Dong*, Zhenbin Fan \\ School of Mathematical Sciences, Yangzhou University, Yangzhou 225002, P. R. China. \\ Communicated by A. Atangana
}

\begin{abstract}
This paper is concerned with a class of boundary value problem of nonlinear fractional differential equation ${ }^{c} D^{\alpha} u(t)-$ $a^{c} D^{\beta} u(t)+f(t, u(t))=0$. This equation may be regarded as an extension of Bagley-Torvik equations. Some new existence and uniqueness results are obtained by using standard Banach contraction principle and Krasnoselskii's fixed point theorem. (C)2017 All rights reserved.
\end{abstract}

Keywords: Fractional derivative, differential equation, boundary value problem. 2010 MSC: 34A08.

\section{Introduction}

Fractional differential equations have gained considerable attention due to their intensive applications in various fields of science such as physics, mechanics, chemistry, engineering, etc. For details see [8, 11, $14,15,18,19]$. There have been a lot of papers devoted to the study of fractional boundary value problems. See, for example, $[2,4,5,9,12,13,17,20]$. For more information to the existence and uniqueness of nonlinear fractional differential equation we refer the reader to $[7,14,19]$ and references therein. For differential equations with Caputo fractional derivatives see $[1,5,6,20]$.

In this paper we study the existence of solutions for boundary value problem of nonlinear fractional differential equations (BVP in short) of the form

$$
\begin{gathered}
{ }^{c} D^{\alpha} u(t)-a^{c} D^{\beta} u(t)+f(t, u(t))=0, \quad 0<t<1, \\
u(0)=u_{0}, \quad u(1)=u_{1},
\end{gathered}
$$

where ${ }^{c} D^{\alpha}$ and ${ }^{c} D^{\beta}$ are Caputo fractional derivatives with $1<\alpha \leqslant 2$ and $1 \leqslant \beta<\alpha, a \in R$ is a constant and $f:[0,1] \times \mathbf{R} \rightarrow \mathbf{R}$ is a given function satisfying some assumptions that will be specified later.

\footnotetext{
${ }^{*}$ Corresponding author

Email addresses: badawi.12@hotmail.com (Badawi Hamza Elbadawi Ibrahim), qxdongyz@outlook.com, qxdong@yzu.edu.cn (Qixiang Dong), zbfan@yzu.edu.cn (Zhenbin Fan)

doi:10.22436/jnsa.010.02.16
} 
Multi-term fractional differential equations have some concrete applications in many fields. However, for a general multi-term fractional differential equation almost no results seems to be known. Only some special cases have been investigated. See, for example, [7, Chapter 8]. In 1984, Bagley and Torvik [3] formulated the mathematical model of the motion of a thin plate in a Newtonian fluid

$$
A y^{\prime \prime}(t)+B^{c} D^{3 / 2} y(t)+C y(t)=f(t),
$$

which is called Bagley-Torvik equation later. Here A, B, and C are certain constants and $f$ is a given function. In [13] Kaufmann and Yao studied the boundary value problem (1.1) with zero bounded conditions involving Riemann-Liouville fractional derivatives, which is a generalization of Bagley-Torvik equation. Existence results were obtained by various fixed point theorems. In [9], the authors studied this problem in Banach spaces.

In this paper we consider the boundary value problem (1.1)-(1.2) with the Caputo fractional derivatives and the boundary values are nonzero. Some sufficient conditions for the existence results are obtained. Banach contraction principle and Krasnoselskii fixed point theorem are employed to deal with this problem. Our results can be regarded as an extension of corresponding results of Bagley-Torvik equation and partially extend the results in [7] and [13].

\section{Preliminaries and lemmas}

In this section we collect some definitions and results which will be used in this paper. Let us denote by $\mathrm{C}([\mathrm{a}, \mathrm{b}], \mathbf{R})$ the Banach space of all continuous functions $u:[a, b] \rightarrow \mathbf{R}$ endowed with supermium norm $\|u\|=\sup \{|u(t)|, t \in[a, b]\}$.

Definition 2.1 ([7]). Let $\alpha>0$ be a fixed number. The Riemann-Liouville fractional integral of order $\alpha>0$ of a function $h:[a, b] \rightarrow \mathbf{R}$ is defined by

$$
I_{a}^{\alpha} h(t)=\frac{1}{\Gamma(\alpha)} \int_{a}^{t}(t-s)^{\alpha-1} h(s) d s, t \in[a, b],
$$

provided the right side is point-wisely defined, where $\Gamma(\cdot)$ denotes the well-known gamma function, i.e., $\Gamma(z)=\int_{0}^{\infty} \mathrm{e}^{-\mathrm{t}} \mathrm{t}^{z-1} \mathrm{dt}$

Definition 2.2 ([7]). Let $\alpha>0$ be fixed and $n=[\alpha]+1$. The Riemann-Liouville fractional derivative of order $\alpha$ of $h:[a, b] \rightarrow \mathbf{R}$ at the point $t$ is defined by

$$
D_{a}^{\alpha} h(t)=\frac{1}{\Gamma(n-\alpha)} \frac{d^{n}}{d t^{n}} \int_{a}^{t}(t-s)^{n-\alpha-1} h(s) d s, t \in[a, b],
$$

provided the right side is point-wisely defined, where $[\alpha]$ denotes the integer part of the real number $\alpha$.

Definition 2.3 ([7]). Let $h:[a, b] \rightarrow \mathbf{R}, \alpha>0$, and $n=[\alpha]+1$. The Caputo fractional derivative of order $\alpha$ of $h$ at the point $t$ is defined by

$$
{ }^{c} D_{a}^{\alpha} h(t)=\frac{1}{\Gamma(n-\alpha)} \int_{a}^{t}(t-s)^{n-\alpha-1} h^{(n)}(s) d s, \quad t \in[a, b],
$$

provided the right side is point-wisely defined. ${ }^{c} \mathrm{D}_{\mathrm{a}}^{\alpha}$ is also called the Caputo fractional differential operator.

For simplicity, when $\mathrm{a}=0$, we denote ${ }^{c} \mathrm{D}_{0}^{\alpha}$ and $\mathrm{I}_{0}^{\alpha}$ by ${ }^{\mathrm{c}} \mathrm{D}^{\alpha}$ and $\mathrm{I}^{\alpha}$, respectively.

Lemma 2.4 ([7]). Let $\alpha>0$ and $\mathrm{m}=[\alpha]+1$. Then the solutions to the equation ${ }^{\mathrm{c}} \mathrm{D}^{\alpha} \mathrm{u}(\mathrm{t})=0$ is given by

$$
\mathrm{u}(\mathrm{t})=\mathrm{c}_{0}+\mathrm{c}_{1} \mathrm{t}+\mathrm{c}_{2} \mathrm{t}^{2}+\cdots+\mathrm{c}_{\mathrm{m}-1} \mathrm{t}^{\mathrm{m}-1},
$$


where $\mathrm{c}_{\mathfrak{i}} \in \mathbf{R}, \mathfrak{i}=0,1,2, \cdots, \mathrm{m}-1$ are some constants. If further assume that $\mathrm{u} \in \mathrm{C}^{\mathrm{m}}([0, \mathrm{~b}] ; \mathbf{R})$, then

$$
\mathrm{I}^{\alpha c} D^{\alpha} u(t)=u(t)+c_{0}+c_{1} t+c_{2} t^{2}+\cdots+c_{m-1} t^{m-1},
$$

for some constants $c_{i} \in \mathbf{R}, i=0,1,2, \cdots, m-1$.

To study the existence of the boundary value problem (1.1)-(1.2), we need to transform the fractional differential equation into an integral equation. We first study the linear version of the problem (1.1)-(1.2).

Lemma 2.5. Suppose that $\mathrm{a} \neq \Gamma(\alpha-\beta+2)$, and $\mathrm{h} \in \mathrm{C}([0,1], \mathbf{R})$ be given. Then the solution $\mathrm{u} \in \mathrm{C}([0,1], \mathbf{R})$ of the fractional differential equation

$$
{ }^{c} D^{\alpha} u(t)-a^{c} D^{\beta} u(t)+h(t)=0, \quad 1<\alpha<2,
$$

with the boundary value conditions

$$
\mathfrak{u}(0)=\mathfrak{u}_{0}, \quad \mathfrak{u}(1)=\mathfrak{u}_{1},
$$

satisfies the integral function

$$
u(t)=p(t)+\int_{0}^{1} G_{1}(t, s) u(s) d s-\int_{0}^{1} G_{2}(t, s) h(s) d s,
$$

where

$$
\begin{aligned}
& p(t)=\frac{\Gamma(\alpha-\beta+2)\left[\Gamma(\alpha-\beta+1)\left(u_{1}-u_{0}\right)+a u_{0}\right]}{[\Gamma(\alpha-\beta+2)-a] \Gamma(\alpha-\beta+1)} t \\
& +\frac{\Gamma(\alpha-\beta+1)\left(u_{0}-u_{1}\right) a-a^{2} u_{0}}{[\Gamma(\alpha-\beta+2)-a] \Gamma(\alpha-\beta+1)} t^{\alpha-\beta+1} \\
& -\frac{a u_{0}}{\Gamma(\alpha-\beta+1)} t^{\alpha-\beta}+u_{0} \text {, } \\
& \mathrm{G}_{1}(\mathrm{t}, \mathrm{s})=\frac{\mathrm{a}}{\Gamma(\alpha-\beta)} \begin{cases}\frac{\mathrm{at} \mathrm{t}^{\alpha-\beta+1}-\Gamma(\alpha-\beta+2) t}{\Gamma(\alpha-\beta+2)-\alpha}(1-s)^{\alpha-\beta-1} & 0 \leqslant s<t \leqslant 1, \\
+(t-s)^{\alpha-\beta-1}, & \\
\frac{a t^{\alpha-\beta+1}-\Gamma(\alpha-\beta+2) t}{\Gamma(\alpha-\beta+2)-a}(1-s)^{\alpha-\beta-1}, & 0 \leqslant \mathrm{t}<\mathrm{s} \leqslant 1,\end{cases}
\end{aligned}
$$

and

$$
G_{2}(t, s)=\frac{1}{\Gamma(\alpha)}\left\{\begin{array}{cl}
\frac{a t^{\alpha-\beta+1}-\Gamma(\alpha-\beta+2) t}{\Gamma(\alpha-\beta+2)-a}(1-s)^{\alpha-1} & 0 \leqslant s<t \leqslant 1, \\
+(t-s)^{\alpha-1}, & \\
\frac{a t^{\alpha-\beta+1}-\Gamma(\alpha-\beta+2) t}{\Gamma(\alpha-\beta+2)-a}(1-s)^{\alpha-1}, & 0 \leqslant t<s \leqslant 1 .
\end{array}\right.
$$

Proof. Since $1<\alpha \leqslant 2$, by Lemma 2.4 ,

$$
I^{\alpha c} D^{\alpha} u(t)=u(t)+c_{1}+c_{2} t
$$

for some constants $c_{1}$ and $c_{2}$ and $t \in[0,1]$. Applying the operator $I^{\alpha}$ to both side of (2.1), one obtains that

$$
I^{\alpha c} D^{\alpha} u(t)=a I^{\alpha c} D^{\beta} u(t)-I^{\alpha} h(t),
$$

for $t \in[0,1]$. Due to the property of fractional integral and Lemma 2.4,

$$
\begin{aligned}
\mathrm{I}^{\alpha c} D^{\beta} u(t) & =I^{\alpha-\beta}\left(I^{\beta c} D^{\beta} u(t)\right) \\
& =I^{\alpha-\beta}\left(u(t)+c_{1}+c_{2} t\right) \\
& =I^{\alpha-\beta} u(t)+\frac{c_{1} t^{\alpha-\beta}}{\Gamma(\alpha-\beta+1)}+\frac{c_{2} t^{\alpha-\beta+1}}{\Gamma(\alpha-\beta+2)} .
\end{aligned}
$$


So we have

$$
u(t)+c_{1}+c_{2} t=a I^{\alpha-\beta} u(t)+\frac{a c_{1} t^{\alpha-\beta}}{\Gamma(\alpha-\beta+1)}+\frac{a c_{2} t^{\alpha-\beta+1}}{\Gamma(\alpha-\beta+2)}-I^{\alpha} h(t),
$$

for $t \in[0,1]$. Then using the boundary value condition (2.2) we get that $c_{1}=-u_{0}$ and

$$
\begin{aligned}
c_{2}= & \frac{\Gamma(\alpha-\beta+2)\left[\mathfrak{u}_{0}-u_{1}+a I^{\alpha-\beta} u(1)-I^{\alpha} h(1)\right]}{\Gamma(\alpha-\beta+2)-a} \\
& -\frac{\Gamma(\alpha-\beta+2) a u_{0}}{[\Gamma(\alpha-\beta+2)-a] \Gamma(\alpha-\beta+1)} .
\end{aligned}
$$

Substituting the value of $c_{1}$ and $c_{2}$ into (2.3), we obtain the desired result, and the lemma is thus proved.

It is easy to see that $G_{2}$ is continuous, and therefore bounded on $[0,1] \times[0,1]$, while $G_{1}$ is unbounded since $\alpha-\beta-1<0$. However, $\int_{0}^{1} G_{1}(t, s)$ ds is uniformly bounded for $t \in[0,1]$. This is because

$$
\begin{aligned}
\int_{0}^{1}\left|G_{1}(t, s)\right| d s \leqslant & \frac{|a|\left[\Gamma(\alpha-\beta+2) t+|a| t^{\alpha-\beta+1}\right]}{\Gamma(\alpha-\beta)|\Gamma(\alpha-\beta+2)-a|} \int_{0}^{1}(1-s)^{\alpha-\beta-1} d s \\
& +\frac{|a|}{\Gamma(\alpha-\beta)} \int_{0}^{t}(t-s)^{\alpha-\beta-1} d s \\
\leqslant & \frac{|a|}{\Gamma(\alpha-\beta+1)}\left(\frac{[\Gamma(\alpha-\beta+2)+|a|]}{|\Gamma(\alpha-\beta+2)-a|}+1\right),
\end{aligned}
$$

for all $t \in[0,1]$. So we denote by

$$
M_{1}=\max _{0 \leqslant t \leqslant 1} \int_{0}^{1}\left|G_{1}(t, s)\right| d s,
$$

and

$$
M_{2}=\max _{0 \leqslant t \leqslant 1} \int_{0}^{1}\left|G_{2}(t, s)\right| d s .
$$

Since $p$ is a polynomial type function, it is obviously continuous and bounded on the interval $[0,1]$. Let

$$
M_{3}=\max _{0 \leqslant t \leqslant 1}|p(t)| .
$$

Theorem 2.6 (Krasnoselskii's fixed point theorem [16]). Let $M$ be a closed, bounded, convex and nonempty subset of a Banach space X. Let A, B be the operators such that

(i) $\mathrm{Ax}+\mathrm{By} \in \mathrm{M}$ whenever $\mathrm{x}, \mathrm{y} \in \mathrm{M}$;

(ii) A is compact and continuous;

(iii) $\mathrm{B}$ is a contraction mapping.

Then there exists $z \in M$ such that $z=A z+B z$.

Theorem 2.7 (Leray-Schauder alternative [10]). Let X be a Banach space, $\mathrm{C} \subset \mathrm{X}$ be a closed, convex subset of $\mathrm{X}, \mathrm{U}$ an open subset of $\mathrm{C}$ and $0 \in \mathrm{U}$. Suppose that $\mathrm{T}: \overline{\mathrm{U}} \rightarrow \mathrm{C}$ is a continuous, compact (that is, $\mathrm{T}(\overline{\mathrm{U}})$ is a relatively compact subset of C) map. Then either

(i) Thas a fixed point in $\overline{\mathrm{U}}$, or

(ii) there is $a \mathrm{u} \in \partial \mathrm{U}$ and $\lambda \in(0,1)$ with $u=\lambda \mathrm{T}(\mathrm{u})$. 


\section{Existence results}

In this section, we study the existence of solutions to BVP (1.1)-(1.2). We begin with the definition of solutions to this problem.

Definition 3.1. A continuous function $u:[0,1] \rightarrow \mathbf{R}$ is said to be a solution to (1.1)-(1.2), if $u$ satisfies

$$
u(t)=p(t)+\int_{0}^{1} G_{1}(t, s) u(s) d s-\int_{0}^{1} G_{2}(t, s) f(s, u(s)) d s,
$$

for $t \in[0,1]$.

For the forthcoming analysis, we need the following hypotheses.

(H1) $f:[0,1] \times \mathbf{R} \rightarrow \mathbf{R}$ is continuous.

(H2) There exists a constant $k>0$ such that

$$
|f(t, u)-f(t, v)| \leqslant k|u-v|,
$$

for all $u, v \in \mathbf{R}$ and $t \in[0,1]$.

(H3) There exists a continuous function $\mu:[0,1] \rightarrow \mathbf{R}^{+}$such that

$$
|f(t, u)| \leqslant \mu(t),
$$

for all $(t, u) \in[0,1] \times \mathbf{R}$.

(H4) There exist functions $\phi \in \mathrm{C}\left([0,1], \mathbf{R}^{+}\right)$and $\psi: \mathbf{R} \rightarrow \mathbf{R}^{+}$nondecreasing such that

$$
|f(t, u)| \leqslant \phi(t) \psi(|u|),
$$

for each $(t, u) \in[0,1] \times \mathbf{R}$.

We first prove an existence result in the case that $f$ satisfies the Lipschitz condition.

Theorem 3.2. Suppose that the condition $(\mathrm{H} 1)$ and $(\mathrm{H} 2)$ are satisfied. If

$$
\mathrm{M}_{1}+\mathrm{kM}_{2}<1,
$$

then the BVP (1.1)-(1.2) has a unique solution in $\mathrm{C}([0,1], \mathbf{R})$.

Proof. Define an operator $\mathrm{T}: \mathrm{C}([0,1], \mathbf{R}) \rightarrow \mathrm{C}([0,1], \mathbf{R})$ by

$$
T u(t)=p(t)+\int_{0}^{1} G_{1}(t, s) u(s) d s-\int_{0}^{1} G_{2}(t, s) f(s, u(s)) d s,
$$

for $u \in C([0,1], \mathbf{R})$ and $t \in[0,1]$. Then $T$ is well-defined and $u \in C([0,1], \mathbf{R})$ is a solution to the BVP (1.1)-(1.2), if and only if $u$ is a fixed point of $T$. We prove that $T$ has a unique fixed point by Banach contraction principle. In fact, take $u, v \in C([0,1], \mathbf{R})$ arbitrary. Then due to $(\mathrm{H} 2)$, we have

$$
\begin{aligned}
|\mathrm{Tu}(\mathrm{t})-\mathrm{T} v(\mathrm{t})| \leqslant & \int_{0}^{1}\left|\mathrm{G}_{1}(\mathrm{t}, \mathrm{s})\right| \mathrm{u}(\mathrm{s})-v(\mathrm{~s}) \mid \mathrm{ds} \\
& +\int_{0}^{1}\left|\mathrm{G}_{2}(\mathrm{t}, \mathrm{s})\right||\mathrm{f}(\mathrm{s}, \mathrm{u}(\mathrm{s}))-\mathrm{f}(\mathrm{s}, v(\mathrm{~s}))| \mathrm{d} s \\
\leqslant & \|\mathrm{u}-v\| \int_{0}^{1}\left|\mathrm{G}_{1}(\mathrm{t}, \mathrm{s})\right| \mathrm{d} s+\mathrm{k} \int_{0}^{1}\left|\mathrm{G}_{2}(\mathrm{t}, \mathrm{s})\right||\mathrm{u}(\mathrm{s})-v(\mathrm{~s})| \mathrm{d} s \\
& \leqslant\left(M_{1}+\mathrm{kM}_{2}\right)\|\mathrm{u}-v\|,
\end{aligned}
$$


for $t \in[0,1]$, and hence

$$
\|T u-T v\| \leqslant\left(M_{1}+k M_{2}\right)\|u-v\| .
$$

The assumption (3.1) shows that $\mathrm{T}$ is a contraction. By Banach contraction principle, $\mathrm{T}$ has a unique fixed point in $C([0,1], \mathbf{R})$, which is the unique solution to the BVP (1.1)-(1.2).

Next we consider the case that $f$ is uniformly bounded w.r.t. the second variable and prove an existence result by employing the Krasnoselskii's fixed point theorem.

Theorem 3.3. Suppose that $(\mathrm{H} 1)$ and $(\mathrm{H} 3)$ are satisfied. If $\mathrm{M}_{1}<1$, then the BVP (1.1)-(1.2) has at least one solution in $\mathrm{C}([0,1], \mathbf{R})$.

Proof. We define operators $E$ and $S$ from $C([0,1], \mathbf{R})$ into itself by

$$
E u(t)=\int_{0}^{1} G_{1}(t, s) u(s) d s+p(t),
$$

and

$$
\mathrm{Su}(\mathrm{t})=-\int_{0}^{1} \mathrm{G}_{2}(\mathrm{t}, \mathrm{s}) \mathrm{f}(\mathrm{s}, \mathrm{u}(\mathrm{s})) \mathrm{ds},
$$

for $u \in C([0,1], \mathbf{R})$ and $t \in[0,1]$. It is easy to verify that $E$ and $S$ are continuous on $C([0,1], \mathbf{R})$ by Lebesgue's dominated convergence theorem.

Let $\|\mu\|=\max _{0 \leqslant t \leqslant 1}|\mu(t)|$. Since $M_{1}<1$, we can take $r>0$ large enough such that

$$
M_{1}+\frac{M_{2}\|\mu\|+M_{3}}{r}<1
$$

Then we have

$$
r M_{1}+M_{2}\|\mu\|+M_{3}<r .
$$

Set $B_{r}=\{u \in C([0,1], \mathbf{R}):\|u\| \leqslant r\}$. Then $B_{r}$ is a nonempty bounded closed convex subset in $C([0,1], \mathbf{R})$. For any $u, v \in B_{r}$ and $t \in[0,1]$, we have

$$
\begin{aligned}
|E u(t)| & \leqslant \int_{0}^{1}\left|G_{1}(t, s)\right||u(s)| d s+|p(t)| \\
& \leqslant r \int_{0}^{1}\left|G_{1}(t, s)\right| d s+M_{3} \leqslant r M_{1}+M_{3}, \\
|S v(t)| & \leqslant \int_{0}^{1}\left|G_{2}(t, s)\right||f(s, v(s))| d s \\
& \leqslant \int_{0}^{1}\left|G_{2}(t, s)\right||\mu(s)| d s \leqslant M_{2}\|\mu\| .
\end{aligned}
$$

So $|E u(t)+S v(t)| \leqslant|E u(t)|+|S u(t)| \leqslant M_{1} r+M_{2}\|\mu\|+M_{3}$, and hence

$$
\|E u+S v\| \leqslant r M_{1}+M_{2}\|\mu\|+M_{3}<r,
$$

which implies that $E u+S v \in B_{r}$.

On the other hand,

$$
\begin{aligned}
|E u(t)-E v(t)| & \leqslant \int_{0}^{1} G_{1}(t, s)|u(s)-v(s)| d s \\
& \leqslant \int_{0}^{1} G_{1}(t, s)\|u-v\| d s \leqslant M_{1}\|u-v\|
\end{aligned}
$$


for all $u, v \in \mathrm{C}([0,1], \mathbf{R})$ and $t \in[0,1]$. This shows that

$$
\|\mathrm{E} u-E v\| \leqslant M_{1}\|u-v\|,
$$

for all $u, v \in C([0,1], \mathbf{R})$, i.e., $E$ is a contraction since $M_{1}<1$.

Now we prove that $S$ is a compact operator. Take any bounded subset $B \subset C([0,1], \mathbf{R})$. Then there is a constant $r_{0}>0$ such that $\|u\| \leqslant r_{0}$ for all $u \in B$. Similar to the proof of the inequality (3.2) we can prove that $S B$ is bounded. We now prove that $S B$ is also equicontinuous. In fact, take $t_{1}, t_{2} \in[0,1]$ with $0 \leqslant t_{1}<t_{2} \leqslant 1$ and $u \in B$ arbitrary, we have

$$
\begin{aligned}
\left|S u\left(t_{2}\right)-S u\left(t_{1}\right)\right|= & \left|\int_{0}^{1} G_{2}\left(t_{2}, s\right) f(s, u(s)) d s-\int_{0}^{1} G_{2}\left(t_{1}, s\right) f(s, u(s)) d s\right| \\
\leqslant & \frac{1}{\Gamma(\alpha)} \mid \int_{0}^{1} \frac{a t_{2}^{\alpha-\beta+1}-\Gamma(\alpha-\beta+2) t_{2}}{\Gamma(\alpha-\beta+2)-a}(1-s)^{\alpha-1} f(s, u(s)) d s \\
& -\int_{0}^{1} \frac{a t_{1}^{\alpha-\beta+1}-\Gamma(\alpha-\beta+2) t_{1}}{\Gamma(\alpha-\beta+2)-a}(1-s)^{\alpha-1} f(s, u(s)) d s \mid \\
& +\frac{1}{\Gamma(\alpha)} \mid \int_{0}^{t_{2}}\left(t_{2}-s\right)^{\alpha-1} f(s, u(s)) d s \\
& -\int_{0}^{t_{1}}\left(t_{1}-s\right)^{\alpha-1} f(s, u(s)) d s \mid \\
\leqslant & \frac{\Gamma(\alpha-\beta+2)\left|t_{2}-t_{1}\right|+|a|\left|t_{2}^{\alpha-\beta+1}-t_{1}^{\alpha-\beta+1}\right|}{\Gamma(\alpha)|\Gamma(\alpha-\beta+2)-a|} \int_{0}^{1}(1-s)^{\alpha-1}|f(s, u(s))| d s \\
& +\frac{1}{\Gamma(\alpha)} \int_{0}^{t_{1}}\left|\left(t_{2}-s\right)^{\alpha-1}-\left(t_{1}-s\right)^{\alpha-1}\right||f(s, u(s))| d s \\
& +\frac{1}{\Gamma(\alpha)} \int_{t_{1}}^{t_{2}}\left(t_{2}-s\right)^{\alpha-1}|f(s, u(s))| d s \\
\leqslant & \frac{\Gamma(\alpha-\beta+2)\left|t_{2}-t_{1}\right|+|a|\left|t_{2}^{\alpha-\beta+1}-t_{1}^{\alpha-\beta+1}\right|}{\Gamma(\alpha+1)|\Gamma(\alpha-\beta+2)-a|} \| \mu \mid \\
& +\frac{1}{\Gamma(\alpha+1)}\left[\left(t_{2}^{\alpha}-t_{1}^{\alpha}\right)+2\left(t_{2}-t_{1}\right)^{\alpha}\right]\|\mu\| .
\end{aligned}
$$

It is easy to see that $\left|S u\left(t_{2}\right)-S u\left(t_{1}\right)\right| \rightarrow 0$ as $t_{2}-t_{1} \rightarrow 0$ and the convergence is independent to $u \in B$. This means that $S B$ is equicontinuous. So $S B$ is compact in $C([0,1], \mathbf{R})$, by Ascoli-Arzela theorem, for each bounded subset $B \subset C([0,1], \mathbf{R})$, i.e., $S$ is compact. Now we apply Krasnoselskii's fixed point theorem (Theorem 2.6) to the operators $E$ and $S$ to get that there exists at least a $u \in B_{r}$ such that $u=E u+S u$, which is a solution to the BVP (1.1)-(1.2) and the proof is completed.

Theorem 3.4. Suppose that (H1) and (H4) are satisfied. If

$$
M_{1}+M_{2}\|\phi\| \limsup _{r \rightarrow \infty} \frac{\psi(r)}{r}<1,
$$

then the BVP (1.1)-(1.2) has at least one solution on $[0,1]$.

Proof. We first observe that the operator $\mathrm{T}: \mathrm{C}([0,1], \mathbf{R}) \rightarrow \mathrm{C}([0,1], \mathbf{R})$ is continuous. We now prove that $T$ is a compact operator. For any bounded subset $W \in C([0,1], \mathbf{R})$ there is a positive number $\rho$ such that $W \subset B_{\rho}=\{u \in C([0,1], \mathbf{R}):\|u\| \leqslant \rho\}$. Then $B_{\rho}$ is a closed convex and bounded subset in $C([0,1], \mathbf{R})$. For each $u \in B_{\rho}$, we have

$$
|\mathrm{Tu}(\mathrm{t})| \leqslant \int_{0}^{1}\left|\mathrm{G}_{1}(\mathrm{t}, \mathrm{s})\right||\mathrm{u}(\mathrm{s})| \mathrm{d} s+\int_{0}^{1}\left|\mathrm{G}_{2}(\mathrm{t}, \mathrm{s})\right||\mathrm{f}(\mathrm{s}, \mathrm{u}(\mathrm{s}))| \mathrm{d} s+|\mathrm{p}(\mathrm{t})|
$$




$$
\begin{aligned}
& \leqslant \int_{0}^{1}\left|G_{1}(t, s)\right|\|u\| d s+\int_{0}^{1}\left|G_{2}(t, s)\right| \phi(s) \psi(\|u\|) d s+M_{3} \\
& \leqslant M_{1}\|u\|+M_{2}\|\phi\| \psi(\|u\|)+M_{3} \\
& \leqslant M_{1} \rho+M_{2}\|\phi\| \psi(\rho)+M_{3},
\end{aligned}
$$

and hence $\|T u\| \leqslant M_{1} \rho+M_{2}\|\phi\| \psi(\rho)+M_{3}$. This means that $\mathrm{TB}_{\rho}$ is uniformly bounded. Now let $u \in B_{\rho}$ arbitrary and $t_{1}, t_{2} \in[0,1]$ with $t_{1}<t_{2}$. Then we have

$$
\begin{aligned}
\left|T u\left(t_{2}\right)-T u\left(t_{1}\right)\right| \leqslant & \left|\int_{0}^{1}\left(G_{1}\left(t_{2}, s\right)-G_{1}\left(t_{1}, s\right)\right) u(s) d s\right| \\
& +\left|\int_{0}^{1}\left(G_{2}\left(t_{2}, s\right)-G_{2}\left(t_{1}, s\right)\right) f(s, u(s)) d s\right|+\left|p\left(t_{2}\right)-p\left(t_{1}\right)\right| \\
\leqslant & \frac{|a|\left|a\left(t_{2}^{\alpha-\beta+1}-t_{1}^{\alpha-\beta+1}\right)+\Gamma(\alpha-\beta+2)\left(t_{1}-t_{2}\right)\right|}{\Gamma(\alpha-\beta)|\Gamma(\alpha-\beta+2)-a|} \int_{0}^{1}(1-s)^{\alpha-\beta-1}|u(s)| d s \\
& +\frac{|a|}{\Gamma(\alpha-\beta)}\left|\int_{0}^{t_{2}}\left(t_{2}-s\right)^{\alpha-\beta-1} u(s) d s-\int_{0}^{t_{1}}\left(t_{1}-s\right)^{\alpha-\beta-1} u(s) d s\right| \\
& +\frac{\left|a\left(t_{2}^{\alpha-\beta+1}-t_{1}^{\alpha-\beta+1}\right)+\Gamma(\alpha-\beta+2)\left(t_{1}-t_{2}\right)\right|}{\Gamma(\alpha)|\Gamma(\alpha-\beta+2)-a|} \int_{0}^{1}(1-s)^{\alpha-1}|f(s, u(s))| d s \\
& +\frac{1}{\Gamma(\alpha)} \mid \int_{0}^{t_{2}}\left(t_{2}-s\right)^{\alpha-1} f(s, u(s)) d s-\int_{0}^{t_{1}}\left(t_{1}-s\right)^{\alpha-1} f(s, u(s) d s \mid \\
& +\left|p\left(t_{2}\right)-p\left(t_{1}\right)\right| \\
= & : I_{1}+I_{2}+I_{3}+I_{4}+I_{5} .
\end{aligned}
$$

From the hypotheses (H1) and (H4) we can get that

$$
\begin{aligned}
\mathrm{I}_{1} \leqslant & \frac{|\mathrm{a}|\left|\mathrm{a}\left(\mathrm{t}_{2}^{\alpha-\beta+1}-\mathrm{t}_{1}^{\alpha-\beta+1}\right)+\Gamma(\alpha-\beta+2)\left(\mathrm{t}_{1}-\mathrm{t}_{2}\right)\right|}{\Gamma(\alpha-\beta)|\Gamma(\alpha-\beta+2)-\mathrm{a}|} \mid\|\mathrm{u}\| \int_{0}^{1}(1-s)^{\alpha-\beta-1} \mathrm{ds} \\
\leqslant & \frac{\rho|\mathrm{a}|\left|\mathrm{a}\left(\mathrm{t}_{2}^{\alpha-\beta+1}-\mathrm{t}_{1}^{\alpha-\beta+1}\right)+\Gamma(\alpha-\beta+2)\left(\mathrm{t}_{1}-\mathrm{t}_{2}\right)\right|}{\Gamma(\alpha-\beta+1)|\Gamma(\alpha-\beta+2)-\mathrm{a}|}, \\
\mathrm{I}_{2} \leqslant & \frac{|\mathrm{a}|}{\Gamma(\alpha-\beta)}\left[\int_{0}^{\mathrm{t}_{1}}\left|\left(\mathrm{t}_{2}-\mathrm{s}\right)^{\alpha-\beta-1}-\left(\mathrm{t}_{1}-\mathrm{s}\right)^{\alpha-\beta-1}\right||\mathrm{u}(\mathrm{s})| \mathrm{d} s\right. \\
& \left.+\int_{\mathrm{t}_{1}}^{\mathrm{t}_{2}}\left|\left(\mathrm{t}_{2}-\mathrm{s}\right)^{\alpha-\beta-1}\right||\mathrm{u}(\mathrm{s})| \mathrm{ds}\right] \\
\leqslant & \frac{|\mathrm{a}||| \mathrm{u} \|}{\Gamma(\alpha-\beta)}\left[\int_{0}^{\mathrm{t}_{1}}\left|\left(\mathrm{t}_{2}-\mathrm{s}\right)^{\alpha-\beta-1}-\left(\mathrm{t}_{1}-\mathrm{s}\right)^{\alpha-\beta-1}\right| \mathrm{ds}\right. \\
& \left.+\int_{\mathrm{t}_{1}}^{\mathrm{t}_{2}}\left(\mathrm{t}_{2}-\mathrm{s}\right)^{\alpha-\beta-1} \mathrm{ds}\right] \\
\leqslant & \frac{|\mathrm{a}| \rho}{\Gamma(\alpha-\beta+1)}\left[\left|\mathrm{t}_{2}^{\alpha-\beta}-\mathrm{t}_{1}^{\alpha-\beta}\right|+2\left(\mathrm{t}_{2}-\mathrm{t}_{1}\right)^{\alpha-\beta}\right], \\
\mathrm{I}_{3} \leqslant & \frac{\left|\mathrm{a}\left(\mathrm{t}_{2}^{\alpha-\beta+1}-\mathrm{t}_{1}^{\alpha-\beta+1}\right)+\Gamma(\alpha-\beta+2)\left(\mathrm{t}_{1}-\mathrm{t}_{2}\right)\right|}{\Gamma(\alpha)|\Gamma(\alpha-\beta+2)-\mathrm{a}|} \int_{0}^{1}(1-s)^{\alpha-1} \mathrm{ds}\|\phi\| \psi(\|\mathrm{u}\|) \\
\leqslant & \frac{\|\phi\| \psi(\rho)\left|\mathrm{a}\left(\mathrm{t}_{2}^{\alpha-\beta+1}-\mathrm{t}_{1}^{\alpha-\beta+1}\right)+\Gamma(\alpha-\beta+2)\left(\mathrm{t}_{1}-\mathrm{t}_{2}\right)\right|}{\Gamma(\alpha+1)|\Gamma(\alpha-\beta+2)-\mathrm{a}|} .
\end{aligned}
$$


Similar to $\mathrm{I}_{2}$, we have

$$
\begin{aligned}
\mathrm{I}_{4} \leqslant & \frac{1}{\Gamma(\alpha)}\left[\int_{0}^{t_{1}}\left[\left(\mathrm{t}_{2}-s\right)^{\alpha-1}-\left(\mathrm{t}_{1}-s\right)^{\alpha-1} \mathrm{ds}\right] \phi(s) \psi(\|\mathrm{u}\|)\right. \\
& \left.+\int_{\mathrm{t}_{1}}^{\mathrm{t}_{2}}\left(\mathrm{t}_{2}-s\right)^{\alpha-1} \phi(s) \psi(\|\mathrm{u}\|) d s\right] \\
\leqslant & \frac{1}{\Gamma(\alpha)}\left[\int_{0}^{t_{1}}\left[\left(\mathrm{t}_{2}-s\right)^{\alpha-1}-\left(\mathrm{t}_{1}-s\right)^{\alpha-1} \mathrm{ds}\right]\|\phi\| \psi(\rho)\right. \\
& \left.+\int_{\mathrm{t}_{1}}^{\mathrm{t}_{2}}\left(\mathrm{t}_{2}-s\right)^{\alpha-1} \mathrm{ds}\|\phi\| \psi(\rho)\right] \\
\leqslant & \frac{\|\phi\| \psi(\rho)}{\Gamma(\alpha+1)}\left[\left|\mathrm{t}_{2}^{\alpha}-\mathrm{t}_{1}^{\alpha}\right|+2\left(\mathrm{t}_{2}-\mathrm{t}_{1}\right)^{\alpha}\right]
\end{aligned}
$$

Obviously the right sides of the above inequality tends to zero as $t_{2}-t_{1} \rightarrow 0$, and

$$
\lim _{t_{2}-t_{1} \rightarrow 0} I_{5}=\lim _{t_{2}-t_{1} \rightarrow 0}\left|p\left(t_{2}\right)-p\left(t_{1}\right)\right|=0,
$$

since $p$ is a polynomial like function. It follows that

$$
\lim _{t_{2}-t_{1} \rightarrow 0}\left|T u\left(t_{2}\right)-T u\left(t_{1}\right)\right|=0,
$$

and the convergence is independent on $u \in B_{\rho}$, i.e., $\mathrm{TB}_{\rho}$ is equicontinuous. By the Arzela-Ascoli theorem we know that $\mathrm{TB}_{\rho}$ is compact. Therefore, the operator $\mathrm{T}: \mathrm{C}([0,1], \mathbf{R}) \rightarrow \mathrm{C}([0,1], \mathbf{R})$ is completely continuous.

Now, from the condition (3.3), there is a positive number $\mathrm{N}$ such that

$$
M_{1} N+M_{2}\|\phi\| \phi(N)+M_{3}<N .
$$

Let $\mathrm{U}=\{\mathrm{u} \in \mathrm{C}([0,1], \mathbf{R}):\|u\|<N\}$. Then $\mathrm{T}: \overline{\mathrm{U}} \rightarrow \mathrm{C}([0,1], \mathbf{R})$ is completely continuous. Suppose that there exist $\lambda \in(0,1)$ and $u \in \bar{u}$ such that $u=\lambda T u$, then for any $t \in[0,1]$,

$$
\begin{aligned}
|u(t)| & =|\lambda T u(t)| \leqslant|T u(t)| \\
& \leqslant|p(t)|+\left|\int_{0}^{1} G_{1}(t, s) u(s) d s\right|+\mid \int_{0}^{1} G_{2}(t, s) f(s, u(s) d s \mid \\
& \leqslant M_{1}\|u\|+M_{2}\|\phi\| \psi(\|u\|)+M_{3},
\end{aligned}
$$

and hence

$$
\mathrm{N}=\|\mathrm{u}\| \leqslant \mathrm{M}_{1}\|\mathrm{u}\|+\mathrm{M}_{2}\|\phi\| \psi(\|\mathrm{u}\|)+\mathrm{M}_{3}<\mathrm{N}
$$

a contradiction. Therefore, for any $u \in \bar{U}$ and $\lambda \in(0,1), u \neq \lambda T u$. By the Leray Schauder alternative, we deduce that $T$ has at least a fixed point $u \in \bar{U}$, which is a solution to the BVP (1.1)-(1.2), and the proof is completed.

\section{Acknowledgment}

This research was partially supported by the National Natural Science Foundation of China (11571300 and 11271316), the Natural Science Foundation for Universities in Jiangsu Province of China (16KJB110023), the Qing Lan Project of Jiangsu Province of China and High-Level Personnel Support Program of Yangzhou University.

\section{References}

[1] B. Ahmed, S. K. Ntouyas, A. Alsaedi, New existence results for nonlinear fractional differential equations with three-point integral boundary conditions, Adv. Difference Equ., 2011 (2011), 11 pages. 1

[2] T. S. Aleroev, On a boundary value problem for a fractional-order differential operator, (Russian) translated from Differ. Uravn., 34 (1998), 123, Differential Equations, 34 (1998), 126.1 
[3] R. L. Bagley, P. J. Torvik, On the appearance of the fractional derivative in the behavior of real materials, J. Appl. Mech., 51 (1984), 294-298. 1

[4] Z.-B. Bai, H. Lü, Positive solutions for a boundary value problem of nonlinear fractional differential equation, J. Math. Anal. Appl., 311 (2005), 495-505. 1

[5] M. Benchohra, S. Hamani, S. K. Ntouyas, Boundary value problems for differential equations with fractional order and nonlocal conditions, Nonlinear Anal., 71 (2009), 2391-2396. 1

[6] M. Benchohra, N. Hamidi, Fractional order differential inclusions on the half-line, Surv. Math. Appl., 5 (2010), 99-111. 1

[7] K. Diethelm, The analysis of fractional differential equations, An application-oriented exposition using differential operators of Caputo type, Lecture Notes in Mathematics, (2004), Springer-Verlag, Berlin, Springer-Verlag, Berlin, (2010). 1, 1, 2.1, 2.2, 2.3, 2.4

[8] K. Diethelm, A. D. Freed, On the solution of nonlinear fractional-order differential equations used in the modeling of viscoplasticity, Sci. Comput. Chem. Eng., II, Springer Berlin Heidelberg, (1999), 217-224. 1

[9] Q.-X. Dong, G.-X. Wu, J. Li, A boundary value problem for a class of fractional differential equations in Banach spaces, (Chinese) Pure Appl. Math. (Xi'an), 29 (2013), 1-10. 1, 1

[10] A. Granas, J. Dugundji, Fixed point theory, Springer Monographs in Mathematics, Springer-Verlag, New York, (2003). 2.7

[11] N. Heymans, I. Podlubny, Physical interpretation of initial conditions for fractional differential equations with RiemannLiouville fractional derivatives, Rheol. Acta, 45 (2006), 765-772. 1

[12] H. Jafari, V. Daftardar-Gejji, Positive solutions of nonlinear fractional boundary value problems using Adomian decomposition method, Appl. Math. Comput., 180 (2006), 700-706. 1

[13] E. R. Kaufmann, K. D. Yao, Existence of solutions for a nonlinear fractional order differential equation, Electron. J. Qual. Theory Differ. Equ., 2009 (2009), 9 pages. 1, 1

[14] A. A. Kilbas, H. M. Srivastava, J. J. Trujillo, Theory and applications of fractional differential equations, North-Holland Mathematics Studies, Elsevier Science B.V., Amsterdam, (2006). 1

[15] P. A. Kilbas, J. J. Trujillo, Differential equations of fractional order: methods, results and problems, II, Appl. Anal., 81 (2002), 435-493. 1

[16] M. A. Krasnoselskii, Two remarks on the method of successive approximations, (Russian) Uspehi Mat. Nauk (N.S.), 10 (1955), 123-127. 2.6

[17] X.-P. Liu, M. Jia, B.-F. Wu, Existence and uniqueness of solution for fractional differential equations with integral boundary conditions, Electron. J. Qual. Theory Differ. Equ., 2009 (2009), 10 pages. 1

[18] F. Metzler, W. Schick, H. G. Kilian, T. F. Nonnenmacher, Relaxation in filled polymers: A fractional calculus approach, J. Chem. Phys., 103 (1995), 7180-7186. 1

[19] K. S. Miller, B. Ross, An introduction to the fractional calculus and fractional differential equations, A Wiley-Interscience Publication, John Wiley \& Sons, Inc., New York, (1993). 1

[20] S. Ntouyas, Existence results for first order boundary value problems for fractional differential equations and inclusions with fractional integral boundary conditions, J. Fract. Calc. Appl., 3 (2012), 1-14. 1 